\title{
Tecnologia educacional como estratégia integrativa de complementação na formação de estudantes e profissionais da área da saúde: Revisão integrativa
}

\author{
Educational technology as an integrative complementary strategy in the training of students and \\ professionals in the health area: Integrative review
}

La tecnología educativa como estrategia complementaria integradora en la formación de estudiantes y profesionales del área de la salud: Revisión integradora

Recebido: 27/07/2021 | Revisado: 31/07/2021 | Aceito: 31/07/2021 | Publicado: 06/08/2021

\author{
Célio Amoêdo de Melo \\ ORCID: https://orcid.org/0000-0003-4539-9230 \\ Centro Universitário Fibra, Brasil \\ E-mail: celioamoedo@gmail.com \\ Maísa Silva de Sousa \\ ORCID: https://orcid.org/0000-0001-9944-7838 \\ Universidade Federal do Pará, Brasil \\ E-mail:maisasousa@ufpa.br
}

\begin{abstract}
Resumo
O presente estudo visa descrever o conteúdo bibliográfico disponível na literatura acerca do uso das tecnologias educacionais como ferramentas integrativas de complementação na formação de estudantes e profissionais da área da saúde, com vistas a corroborar para a promoção do ensino em saúde e promover alternativas viáveis e acessíveis no desenvolvimento de métodos educacionais em citologia clínica. O estudo compreendeu a primeira etapa de desenvolvimento teórico aplicado à construção de Webtecnologia, mediada a partir de uma revisão bibliográfica do tipo integrativa, de base observacional e de natureza qualitativa, fundamentada por meio de estudos que reiteram temas acerca de tecnologias educacionais como ferramentas para o desenvolvimento profissional na área da saúde. Os resultados encontrados, tratam principalmente da necessidade da mudança na forma de aprendizagem, fundamentado no alcance à adaptação dos novos meios e tecnologias para o sistema de educação superior. De acordo com as tendências observadas no meio de ensino, as mudanças nos requisitos da educação e da sociedade têm sido amplamente discutidas na literatura. A adesão dessas tecnologias deve ampliar o potencial gerador de conhecimento, além do desenvolvimento de estudantes de citologia clínica, revolucionando o papel do profissional e traduzindo novas mudanças na prática do ensino em citologia.
\end{abstract}

Palavras-chave: Blog; Teste de papanicolaou; Educação continuada; Tecnologias educacionais.

\begin{abstract}
This study aims to describe the bibliographic content available in the literature on the use of educational technologies as integrative complementary tools in the training of students and health professionals, with a view to supporting the promotion of health education and promoting viable and accessible alternatives in the development of educational methods in clinical cytology. The study comprised the first stage of theoretical development applied to the construction of Webtechnology, mediated from an integrative bibliographic review, observational and qualitative in nature, based on studies that reiterate themes about educational technologies as tools for the professional development in the health field. The results found mainly deal with the need to change the way of learning, based on the scope for adapting new means and technologies for the higher education system. According to the trends observed in the teaching environment, changes in the requirements of education and society have been widely discussed in the literature. Adherence to these technologies should expand the potential for generating knowledge, in addition to the development of clinical cytology students, revolutionizing the role of the professional and translating new changes in the practice of teaching in cytology. Keywords: Blog; Pap smear test; Continuing education; Educational technologies.

\section{Resumen}

Este estudio tiene como objetivo describir el contenido bibliográfico disponible en la literatura sobre el uso de tecnologías educativas como herramientas integradoras complementarias en la formación de estudiantes y profesionales de la salud, con miras a apoyar la promoción de la educación para la salud y promover alternativas viables y accesibles en el desarrollo. de métodos educativos en citología clínica. El estudio comprendió la primera etapa de desarrollo teórico aplicado a la construcción de tecnologías web, mediado a partir de una revisión bibliográfica integradora, de carácter observacional y cualitativo, a partir de estudios que reiteran temáticas sobre tecnologías educativas como herramientas
\end{abstract}


para el desarrollo profesional en el campo de la salud. Los resultados encontrados versan principalmente sobre la necesidad de cambiar la forma de aprender, a partir del alcance de adaptar nuevos medios y tecnologías para el sistema de educación superior. De acuerdo con las tendencias observadas en el entorno docente, los cambios en los requisitos de la educación y la sociedad han sido ampliamente discutidos en la literatura. La adherencia a estas tecnologías debería ampliar el potencial de generación de conocimiento, además del desarrollo de los estudiantes de citología clínica, revolucionando el rol del profesional y traduciendo nuevos cambios en la práctica de la docencia en citología.

Palabras clave: Blog; Prueba de Papanicolaou; Educación contínua; Tecnologías educativas.

\section{Introdução}

O Câncer de colo de útero (CCU) é considerado um grave problema de saúde pública em todo o mundo, responsável por 250.000 mortes em países de média e baixa renda. Ao longo dos anos, tem-se tornado um foco de preocupação constante, por isso, vários estudos têm demonstrado interesse em minimizar os seus números, apresentando uma variedade de técnicas de vertente epidemiológica, experimental e educacional, que busquem viabilizar e promover uma ação imediata no diagnóstico precoce (Pinheiro et al., 2014; Sanches et al., 2015; Sanches, et al., 2018; Santos, 2017). Os diferentes critérios usados pelos laboratórios no escrutínio das lâminas do teste preventivo do câncer de colo uterino (Papanicolaou) podem ser fatores que acomodam essa ocorrência (Rodrigues et al., 2017).

A ciência responsável por contribuir de maneira significativa com essa problemática é a citologia clínica, fazendo-se presente na grade curricular de diversos cursos da área da saúde. Nota-se um elevado grau de dificuldade de aprendizado, pois a alta variação morfológica e a necessidade de refinada interpretação das alterações nas células visualizadas, dificultam o reconhecimento, com um destaque especial às células endocervicais, devido a sua característica de apresentar uma variedade de imagens quando observadas em lâminas. Ainda, devido à sua grande fragilidade se desintegram facilmente, dificultando a identificação para observadores menos experientes (Peuker, 2017; Carvalho, 2020).

A citologia clínica também é a ciência que contribui com os achados morfológicos no rastreamento de determinadas doenças, que incluem o CCU, cujo diagnóstico se dá por meio de comparações de imagens celulares microscópicas préestabelecidas segundo o sistema internacional de classificação Bethesda (Siqueira et al., 2014). Nessa perspectiva, deve-se priorizar a atenção à uma formação competente e especializada, de profissionais capacitados que compreendam a necessidade de estabelecer um diagnóstico preciso e que reconheçam as dificuldades na leitura e interpretação da morfologia celular. Diante da carência de protocolos, faz-se necessária a atenção ao processo de ensino e aprendizagem na formação desses profissionais (Calumby, 2020).

Ao longo dos anos, tem-se dada certa atenção aos modelos aplicados no desenvolvimento do conhecimento e da aprendizagem. O processo de compreensão do ensino e da aprendizagem, enquanto propriedade fundamental à formação humana, coexiste de forma mútua, apresentando elementos distintos que se caracterizam como propriedades essenciais tanto do indivíduo, quanto da disponibilidade dos produtos decorrentes do meio em que ele é submetido (Alexandre, 2010). Com ênfase na relação entre o indivíduo e o objeto de conhecimento, diversas teorias de como o processo de formação do homem ocorre são discutidas, a fim de remodelar uma conjuntura específica de ensino, a partir da distinção das principais posturas teóricometodológicas aplicadas na atualidade (Palangana, 1998, Santos, Kaulfuss, 1997).

A aprendizagem é caracterizada como um processo decorrente da transformação do comportamento adquirido, a partir de um intermédio específico das experiências coletivas e individuais, sob aspectos neurológicos, emocionais, sociais e ambientais que, em conjunto com as concepções e comportamentos que o sujeito considera adequado, promove uma gama de experiências que evidenciam a forma do indivíduo se comportar diante de uma situação. Nessa perspectiva, o produto final da aprendizagem pode ser observado a partir da mudança comportamental do indivíduo, aplicado na reprodutibilidade do conhecimento adquirido (Rego, 2005; Domingos, 2017; Gasparin, 2015). 
Considerando as possibilidades de aprendizagem no cenário da teoria e prática profissional, na área da saúde, sob seus princípios e diretrizes, há um conjunto de requisitos fundamentais para a identificação das necessidades da saúde e cuidado centrado no paciente. De modo a promover uma aprendizagem significativa e concatenada no processo contínuo de formação, diversos modelos de ensino com enfoque na evolução técnica do profissional, favorecendo seu processo interno de construção pessoal e profissional. Em virtude disso, muitos educadores têm procurado compreender e responder os desafios propostos na busca por métodos eficazes de ensino que compreendem as necessidades da contemporaneidade (Nalon, et al., 2019; Lacanallo, 2019; Ghiraldelli, 2000).

Como instrumento de educação, a tecnologia tem sido uma alternativa eficaz na promoção do ensino em saúde. A partir de sua utilização como meio inovador de ensino, as novas tecnologias promovem o acesso irrestrito e continuado ao conteúdo, permitindo uma dinamicidade fundamental para a construção de novos conhecimentos. Em consequência da evolução continuada e da produção de conteúdo, o surgimento dessas tecnologias, tem proporcionado ao longo dos anos, muitas mudanças sociais, flexíveis e personalizadas nas relações de ensino-aprendizagem (Malta-Rocha, Ferreira, Fonseca, 2019 Lakatos; Marconi, 2001).

A citologia clínica, nessa perspectiva, com ênfase na identificação morfológica de estruturas celulares, exige uma compreensão fundamentada em sistemas de ensino organizados e integrados, que viabilizem uma aprendizagem substancial, associada à manipulação adequada do método de ensino e do produto didático ideal para a transmissão do conhecimento. Acredita-se então, que deve ser estimulada a interação entre a estrutura prévia cognitiva do indivíduo e o conteúdo de aprendizagem, a fim de propiciar um processo de ressignificação mútua do núcleo do conhecimento adquirido, crucial para desenvolvimento das habilidades técnicas do profissional (Tacca, Branco, 2018; Mortimer, Chagas, Alvarenga, 1998).

Desse modo, é de fundamental importância a compreensão das dificuldades encontradas durante a formação do profissional, em meio aos métodos de ensino aplicados com ênfase na transmissão do conhecimento, uma vez que, conforme as necessidades se estabelecem no âmbito laboral, a prática pode exigir uma formação especializada, com enfoque na resolução de problemas, e a fim de superá-las, as tecnologias apresentam-se como um modelo extremamente eficaz nesse quesito (Daroda, 2012). Portanto, o presente estudo visa descrever o conteúdo bibliográfico disponível na literatura acerca do uso das tecnologias educacionais como ferramentas integrativas de complementação na formação de estudantes e profissionais da área da saúde, com vistas a incentivar a promoção do ensino em saúde e promover alternativas viáveis e acessíveis no desenvolvimento de métodos educacionais em citologia clínica

\section{Metodologia}

Trata-se de uma revisão bibliográfica do tipo integrativa, de base observacional e de natureza qualitativa, fundamentada por meio de estudos que reiteram temas acerca de tecnologias educacionais como ferramentas para o desenvolvimento profissional na área da saúde.

A estruturação da busca seguiu critérios previamente estabelecidos, com uma perspectiva de enfoque e delineamento de execução da proposta inicial a partir da: (1) formulação da pergunta norteadora; (2) elaboração dos critérios de inclusão e exclusão; (3) definição criteriosa dos filtros de pesquisa; (4) construção de instrumentos para a coleta de dados; (5) consulta, busca e seleção dos Descritores; (6) avaliação e análise dos artigos selecionados; (7) determinação dos estudos a serem utilizados no produto final; (8) interpretação e apresentação dos resultados obtidos, onde foram refinados os dados de acordo com parâmetros selecionados previamente e apresentados juntamente, conforme critério do autor. As bases de dados utilizadas no levantamento do material de estudo foram as plataformas de busca: Medical Literature Analysis and Retrieval System Online (Medline), Scientific Eletronic Library Online (SciELO), Literatura Latino-Americana e do Caribe em Ciências da Saúde (LILACS). 
As palavras-chave utilizadas para triagem e busca dos artigos foram: Blog; Educação Continuada; Teste de Papanicolaou; Tecnologias Educacionais. De modo a apurar os artigos com um viés de busca mais específico, determinou-se como critério de inclusão o período de 2010-2021, com enfoque em estudos realizados acerca do tema proposto, com proposta de inovação, que impactasse a comunidade acadêmica a partir da promoção de novas alternativas de ensino. Ainda, foram utilizados somente estudos disponíveis na íntegra; indexados nas bases de dados; publicados em revistas de alto impacto e disponíveis nos idiomas português, inglês e espanhol. Sob a outra perspectiva, foram rejeitados artigos publicados antes do período determinado, em formato de carta, editoração, short communication ou semelhantes. A Figura 1 detalha o processo de desencadeamento técnico para elaboração metodológica da revisão bibliográfica.

Figura 1: Fluxograma da Revisão da Literatura.

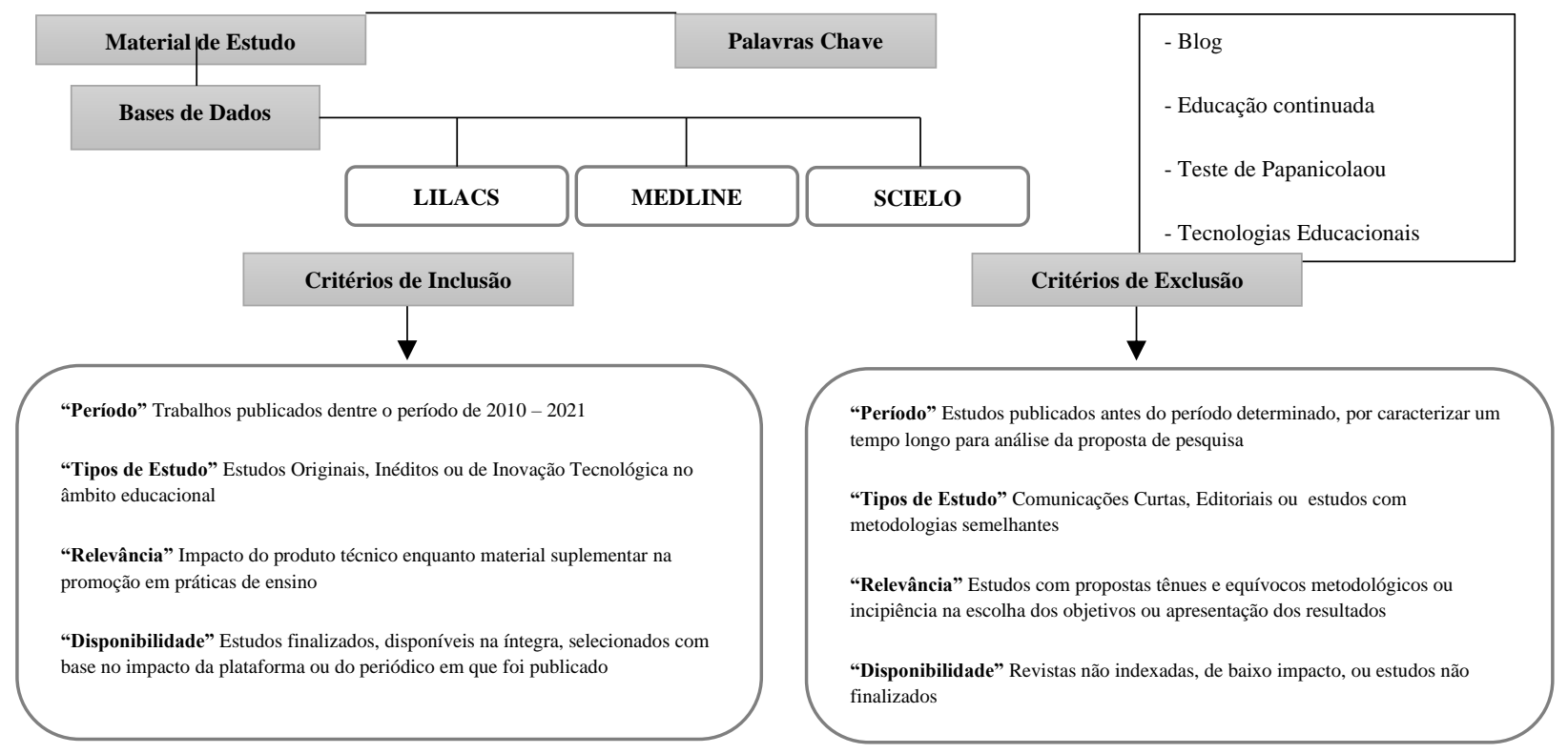

Fonte: Autores.

Quanto aos títulos, foram designados os artigos para avaliação, independente dos resumos. Posteriormente, os resumos dos artigos selecionados foram lidos na integra e forneceram resultados significantes, demonstrado em quadros para melhor compreensão. Ao final desse processo obteve-se todos os estudos de compreensão sob os parâmetros de utilidade, a partir de informações estabelecidas nas seguintes variáveis: i) o autor, e ano de publicação do artigo; (ii) estudos originais; (iii) tecnologias educacionais utilizadas; (iv) contribuição para a comunidade científica; (v) impacto e relevância dos resultados apresentados no estudo. Os resultados foram apresentados a partir da construção de quadros sinópticos, contendo os aspectos de: título do estudo, autores e ano de publicação, periódico, síntese e contribuições.

\section{Resultados e Discussão}

Foram identificados, após busca sistematizada, o total de 17 estudos relacionados com a aplicação de tecnologias educacionais como ferramentas integrativas e complementares para o desenvolvimento profissional, sendo estes registrados em correspondência quantitativa. Na Figura 2, encontra-se o modelo representativo da busca, seleção e obtenção dos estudos. 
Figura 2: Fluxograma dos estudos obtidos após processo de seleção, por representação quantitativa referente à

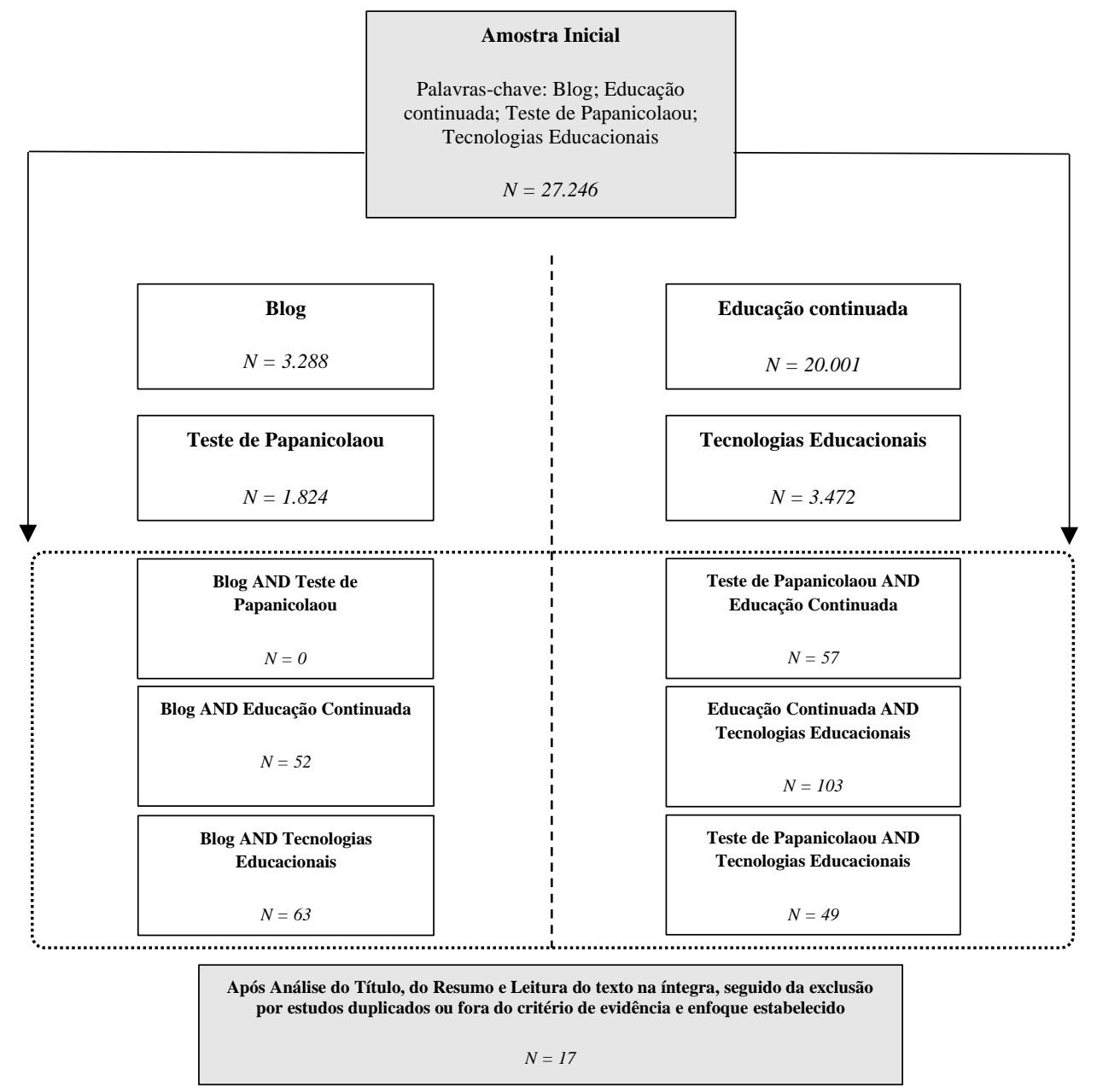

Fonte: Autores.

Após uma análise minuciosa dos artigos obtidos para composição do presente estudo, foram determinadas algumas vertentes de prevalência, às quais propuseram-se delimitar, de forma qualitativa, a necessidade de apresentação dos seus produtos, sendo então escolhidos a partir dos seguintes subtemas; (I) Vivência e desenvolvimento profissional a partir do ensino mediado por tecnologias; (II) Webtecnologia como estratégia integrativa e complementar no processo de ensino-aprendizagem; (III) Educação continuada em Citologia Clínica para capacitação e formação de profissionais em cursos da área da saúde. A escolha destes referidos critérios foi determinada de forma qualitativa, conforme necessidade evidenciada no âmbito de ensino e pesquisa. O Quadro 1, no uso de suas atribuições, busca realizar uma apresentação sob o contexto da instituição, do docente e do discente, acerca da vivência e do desenvolvimento profissional, aplicado a partir da tecnologia, sendo mediada como um produto pedagógico em sala de aula. 
Quadro 1: Vivência e Desenvolvimento Profissional a partir do ensino mediado por tecnologias.

\begin{tabular}{|c|c|c|c|c|c|}
\hline$\#$ & Autores & Periódico & Título & Síntese & Contribuições \\
\hline 1 & $\begin{array}{l}\text { Bowman, MA. } \\
\text { et al, } 2020\end{array}$ & $\begin{array}{l}\text { Journal of Research } \\
\text { on Technology in } \\
\text { Education }\end{array}$ & $\begin{array}{l}\text { Teachers' exposure to } \\
\text { professional development and the } \\
\text { quality of their instructional } \\
\text { technology use: The mediating } \\
\text { role of theachers' value and } \\
\text { ability beliefs }\end{array}$ & $\begin{array}{l}\text { Buscou-se nesse estudo investigar as } \\
\text { relações entre a exposições ao } \\
\text { desenvolvimento profissional, } \\
\text { habilidades e valores dos professores e } \\
\text { qualidade de ensino em integração a } \\
\text { partir do uso de tecnologias } \\
\text { complementares. Sugerindo que esse } \\
\text { desenvolvimento profissional pode ter } \\
\text { maior eficiência quando voltada a } \\
\text { melhorar a valorização do profissional, } \\
\text { além de aprimorar as habilidades } \\
\text { inerentes ao uso da tecnologia }\end{array}$ & $\begin{array}{l}\text { O estudo sugere que há necessidade em mais } \\
\text { trabalho a ser realizado com vistas a } \\
\text { identificar, inicialmente de baixo, diferentes } \\
\text { formas de alcançar os professores, } \\
\text { influenciando na sua forma de repassar o } \\
\text { ensino, com enfoque na ressignificação de } \\
\text { suas crenças e valores. Dessa forma, os } \\
\text { programas de desenvolvimento precisam ser } \\
\text { mais bem estruturados em conjunto com } \\
\text { estratégias adicionais para a melhor adesão à } \\
\text { tecnologia aplicada ao ensino, especialmente } \\
\text { quanto à melhora da percepção da tecnologia } \\
\text { enquanto método de ensino }\end{array}$ \\
\hline 2 & Filho, PA 2011 & $\begin{array}{l}\text { Educação em } \\
\text { Revista }\end{array}$ & $\begin{array}{l}\text { Educação a Distância: uma } \\
\text { abordagem metodológica didática } \\
\text { a partir dos ambientes virtuais }\end{array}$ & $\begin{array}{l}\text { O referido estudo, busca compreender } \\
\text { a exigência pedagógica da Educação a } \\
\text { Distância pelo enfoque técnico frente } \\
\text { às relações metodológicas e didáticas a } \\
\text { partir da compreensão de uma gama de } \\
\text { métodos que viabilizem o processo de } \\
\text { autonomia do indivíduo, durante a sua } \\
\text { formação }\end{array}$ & $\begin{array}{l}\text { Aborda uma nova perspectiva, baseada na } \\
\text { dissociação da postura passiva, receptiva e } \\
\text { dependente da formação do discente, } \\
\text { colocando-o no papel de construtor do próprio } \\
\text { processo de conhecimento, ao passo em que } \\
\text { promove a utilização de novos modelos } \\
\text { pedagógicos com ênfase no uso das } \\
\text { tecnologias educacionais }\end{array}$ \\
\hline 3 & $\begin{array}{l}\text { Modelski, D. et } \\
\text { al, } 2019\end{array}$ & Educação e Pesquisa & $\begin{array}{l}\text { Tecnologias Digitais, formação } \\
\text { docente e práticas pedagógicas }\end{array}$ & $\begin{array}{l}\text { O artigo apresenta os resultados de } \\
\text { pesquisa envolvendo a formação } \\
\text { docente em tempos de acesso contínuo } \\
\text { às redes de acesso virtual e sua } \\
\text { associação com os conhecimentos, } \\
\text { habilidades e atitudes para atuação de } \\
\text { professores no cenário educacional, } \\
\text { influenciado pelo uso de Tecnologias } \\
\text { Digitais }\end{array}$ & $\begin{array}{l}\text { Foram proporcionados elementos } \\
\text { significativos acerca das competências } \\
\text { docentes das demandas nas instituições, no } \\
\text { contexto de comunicação e informação digital. } \\
\text { Dessa forma, foram apresentados indicativos } \\
\text { de que deve ser incentivado o processo de } \\
\text { desenvolvimento de competências por meio da } \\
\text { instrumentação expressa em práticas } \\
\text { educacionais }\end{array}$ \\
\hline 4 & $\begin{array}{l}\text { Santos, SACE. } \\
\text { et al, } 2020\end{array}$ & $\begin{array}{l}\text { Núcleo do } \\
\text { Conhecimento }\end{array}$ & $\begin{array}{l}\text { Uso da Tecnologia na Educação: } \\
\text { Perspectivas e Entraves }\end{array}$ & $\begin{array}{l}\text { O estudo se propõe a refletir acerca da } \\
\text { existência da desarmonia entre os } \\
\text { olhares da ideologia da sistematização } \\
\text { tecnológica, assim como a maneira } \\
\text { como os educadores utilizam-na para a } \\
\text { formação dos discentes, trazendo uma } \\
\text { perspectiva fundamentada no } \\
\text { conhecimento adquirido formulado de } \\
\text { acordo com a adesão às novas } \\
\text { tecnologias }\end{array}$ & $\begin{array}{l}\text { O estudo apresenta a tecnologia com } \\
\text { potencialidade transformadora e um conjunto } \\
\text { com conexões imaginárias que possam } \\
\text { promover efetivamente, novos caminhos mais } \\
\text { eficientes para a aprendizagem. Nesse sentido, } \\
\text { os autores buscam incentivar a construção do } \\
\text { conhecimento e assim, protagonizar o domínio } \\
\text { da tecnologia como meio para potencializar o } \\
\text { aumento das habilidades profissionais }\end{array}$ \\
\hline 5 & $\begin{array}{l}\text { Beluce, AC. et } \\
\text { al, } 2018\end{array}$ & $\begin{array}{l}\text { School and } \\
\text { Educational } \\
\text { Psychology }\end{array}$ & $\begin{array}{l}\text { Learning Strategies Mediated by } \\
\text { Technologies: Use and } \\
\text { Observation of Teachers }\end{array}$ & $\begin{array}{l}\text { O estudo estabelece o desenvolvimento } \\
\text { de uma escala que mede as estratégias } \\
\text { de aprendizagem utilizadas pelos } \\
\text { docentes e as que eles observam sendo } \\
\text { as mais utilizadas pelos alunos, com } \\
\text { vistas a adotar estratégias de acesso à } \\
\text { web, como mídia social ou internet e } \\
\text { associando às evidencias internas para } \\
\text { a delimitação dessa escala, como uma } \\
\text { forma de monitorar o ensino }\end{array}$ & $\begin{array}{l}\text { De modo geral, obteve-se um fluxo dinâmico } \\
\text { de interações diretamente associado a uma } \\
\text { gama diversificada de informações. Foram } \\
\text { observadas necessidades inerentes à revisão a } \\
\text { partir de estratégias meta - cognitivas e os } \\
\text { itens relacionados ao ambiente virtual, } \\
\text { tornando-se fundamental na compreensão do } \\
\text { uso de estratégias inovadoras que permitem a } \\
\text { regulação e o monitoramento do processo } \\
\text { cognitivo de aprendizado das informações }\end{array}$ \\
\hline 6 & $\begin{array}{l}\text { Shaffer, DW. et } \\
\text { al, } 2015\end{array}$ & $\begin{array}{l}\text { Teachers College } \\
\text { Record }\end{array}$ & $\begin{array}{l}\text { Technology and the New } \\
\text { Professionalization of Teaching }\end{array}$ & $\begin{array}{l}\text { O artigo busca tratar acerca do } \\
\text { panorama em vista da mudança da } \\
\text { educação na era digital para o público } \\
\text { amplo, em diferentes áreas e linhas do } \\
\text { conhecimento, a partir da mudança do } \\
\text { papel do docente em um sistema } \\
\text { educacional rico no acesso à tecnologia } \\
\text { e, a fim de fomentar um apoio } \\
\text { significativo no desenvolvimento } \\
\text { social, emocional e intelectual dos } \\
\text { alunos no ambiente de aprendizagem } \\
\text { digital, procurou-se conhecer as } \\
\text { habilidades, conhecimentos e valores } \\
\text { que os docentes necessitarão para } \\
\text { aplica-los na prática profissional }\end{array}$ & $\begin{array}{l}\text { Foi explorada a influência das tecnologias } \\
\text { educacionais no ensino e na preparação de } \\
\text { professores. Os autores Identificaram distintos } \\
\text { métodos de ensino aplicados às tecnologias } \\
\text { digitais por meio da prática de rotina e } \\
\text { trouxeram a discussão de como essas } \\
\text { tecnologias podem assumir diferentes aspectos } \\
\text { nas funções principais do ensino, tanto no } \\
\text { processo de avaliação, quanto na tutoria e na } \\
\text { explicação do conteúdo. Desse modo, } \\
\text { puderam concluir que essas tecnologias podem } \\
\text { permitir avanços diferenciais com enfoque nas } \\
\text { necessidades básicas dos alunos, sendo } \\
\text { necessária uma nova padronização das } \\
\text { relações docente-discente, com base nesse } \\
\text { novo contexto }\end{array}$ \\
\hline
\end{tabular}

Fonte: Autores.

Os resultados identificados após uma análise realizada de forma comparativa, possuem uma vertente de verossimilhança quanto às necessidades da utilização de tecnologias para a melhora da prática de ensino e do aprendizado. Bowman (2020) e 
Beluce, et al (2018), enfatizam em seus respectivos trabalhos, a dificuldade da integração da tecnologia aplicada às barreiras que inviabilizam o desenvolvimento de estratégias eficazes na formação docente. Essas barreiras, dividem-se em critérios de primeira e segunda ordem que, respectivamente, tratam de fatores como cultura institucional, visão e acesso à tecnologia; das crenças de valor e capacidade.

Bowman (2020) trata do desenvolvimento profissional como melhora da capacidade da formação docente, enquanto mediador do processo de ensino aplicado na vivência e no uso de tecnologias no ambiente de ensino. Alguns aspectos, como a forma pela qual a instituição aplica seus métodos de ensino, a visão e a necessidade do docente de adequar-se às normas institucionais, além do acesso mútuo e compartilhado das tecnologias, tanto da parte institucional, profissional ou pessoal, são alguns fatores imprescindíveis com foco à integração dessas tecnologias.

Bowman (2020) e Beluce, et al (2018), atribuem entre si, uma necessidade de promover uma formação continuada de docentes quantos aos ideais pré-estabelecidos na forma de transmissão de conhecimento, de modo a familiarizar os profissionais de ensino e torná-los confiantes quanto à utilização do conteúdo digital como ferramenta complementar na sala de aula. Dessa forma, esses fatores precisam coexistir de forma paralela, para que resulte em um conhecimento tecnológico, pedagógico e de ensino voltado ao treinamento e integração em tecnologia e do conteúdo de aprendizagem digital. Muitos são os desafios atribuídos à implementação das tecnologias como ferramentas de ensino, principalmente quanto à organização e planejamento que corroborem para implementação como um método usual na rotina pedagógica. Assim como descrito por Filho (2011), a produção de materiais avaliativos que sustentem essa prerrogativa, pode ser eficaz no reconhecimento do processo de ensinoaprendizagem, quanto à conscientização do suprimento intelectual a favor do ensino e da ampliação do conhecimento dos discentes.

Principalmente relativo às áreas que exigem um conhecimento aplicado (que se percebe na citologia clínica), é de suma importância a égide do estímulo à busca de um conhecimento minimamente compartimentalizado, no sentido de habilitar o discente a filtrar, comparar, selecionar, avaliar, sintetizar e contextualizar os conhecimentos possíveis. Filho (2011) e Santos (2020) propõe uma reorientação para um ensino compartilhado, participativo e comunitário. Nesse sentido, as tecnologias educacionais se encaixam em um produto de ensino contínuo, contextualizado ao contexto em que se dispõe à própria formação do docente e a formação contínua de profissionais especializados e capacitados para desenvolvimento de técnicas específicas em suas respectivas áreas.

Sobre o contexto da formação institucional na importância da elegibilidade das tecnologias de ensino no ambiente acadêmico, Moldesi, et al (2019) ressalta o uso pedagógico como competência fundamental no auxílio às instituições, sob o apoio aos docentes, o sentido de proporcionar elementos indicativos desejáveis no desenvolvimento de estratégias inovadoras se correlacionam, como já mencionado, às necessidades em linhas de conhecimento aplicado. Nesse sentido, Moldesi, et al (2019) e Shaffer, et al (2015), destacam o termo “instrumentalização" que, se aplica como um aspecto comum quanto ao nível didático expresso em práticas, de modo a acompanhar, em fluxo contínuo, às necessidades de aprendizagem conforme o avanço do cenário profissional, adaptando os profissionais da saúde quanto ao grau de competência exigido.

Nesse contexto, foi possível perceber que a mera adesão à tecnologia como ferramenta educacional, perpassa adoção, seleção e aquisição de competências para o seu uso, e remete muito mais às origens institucionais e à formação do docente. Dessa forma, além de sustentar o enfoque na fluência digital, deve-se centralizar a transposição didática nos alicerces da formação superior, ou seja, no realinhamento dos processos de ensino-aprendizagem, aplicados às necessidades mais atuais. O Quadro 2 busca retratar os artigos selecionados na literatura, com ênfase em tecnologia educacional (Webtecnologia), como estratégia que vise a integração e a complementação técnica de elementos constitutivos do processo de ensino-aprendizagem. 
Quadro 2: Webtecnologia como estratégia integrativa e complementar no processo de ensino-aprendizagem.

\begin{tabular}{|c|c|c|c|c|c|}
\hline$\#$ & Autores & Periódico & Título & Síntese & Contribuições \\
\hline 1 & $\begin{array}{l}\text { JimoyanA. et } \\
\text { al, } 2010\end{array}$ & $\begin{array}{l}\text { Computers - } \\
\text { Education }\end{array}$ & $\begin{array}{l}\text { Designing and implementing an } \\
\text { integrated technological } \\
\text { pedagogical science knowledge } \\
\text { framework for science teachers } \\
\text { professional development }\end{array}$ & $\begin{array}{l}\text { O presente estudo aborda a concepção e a } \\
\text { implementação do conhecimento da teoria } \\
\text { pedagógica a partir de um novo modelo } \\
\text { para o desenvolvimento profissional de } \\
\text { docentes em um sistema integrado de } \\
\text { webtecnologias a partir de abordagem de } \\
\text { aprendizagem autêntica e inovadora }\end{array}$ & $\begin{array}{l}\text { Foram apresentados resultados de interesse } \\
\text { para a comunidade internacional de pesquisa } \\
\text { e pôde oferecer um importante debate sobre } \\
\text { como incentivar a melhora da educação e do } \\
\text { ensino em docentes em diversas áreas das } \\
\text { ciências, com foco na melhora do perfil do } \\
\text { profissional e na integração da tecnologia em } \\
\text { sala de aula para com os discentes }\end{array}$ \\
\hline 2 & $\begin{array}{l}\text { Germani } \\
\text { AAC. et al, } \\
2014\end{array}$ & $\begin{array}{l}\text { Jornal Brasileiro de } \\
\text { TeleSaúde }\end{array}$ & $\begin{array}{l}\text { Avaliação de tecnologias } \\
\text { educacionais digitais para a } \\
\text { formação de promotores de saúde } \\
\text { em uma experiência } \\
\text { interprofissional }\end{array}$ & $\begin{array}{l}\text { O estudo realizou uma pesquisa acerca da } \\
\text { utilização e recursos digitais do tipo } \\
\text { webtecnologia como uma experiência } \\
\text { semipresencial de formação em promoção } \\
\text { da saúde, de aspecto interprofissional com } \\
\text { e sem apoio de tutoria }\end{array}$ & $\begin{array}{l}\text { Foi observada uma maior utilização das } \\
\text { tecnologias ao longo do curso, sendo que dos } \\
\text { discentes que optaram pela tutoria, teve uma } \\
\text { maior frequência de aceso ao blog. Assim, foi } \\
\text { identificado um impacto significativo no } \\
\text { domínio do conhecimento mediado pelos } \\
\text { recursos didáticos }\end{array}$ \\
\hline 3 & $\begin{array}{l}\text { Lima C. et al, } \\
2020\end{array}$ & Educação em Revista & $\begin{array}{l}\text { Plataformas Digitais de } \\
\text { Aprendizagem: uma revisão } \\
\text { integrativa para apoiar a } \\
\text { internacionalização do ensino } \\
\text { superior }\end{array}$ & $\begin{array}{l}\text { Trata-se de uma revisão bibliográfica } \\
\text { integrativa, do tipo exploratória, que } \\
\text { aborda as publicações cientificas } \\
\text { disponíveis na literatura, envolvendo } \\
\text { tecnologia de informação com enfoque às } \\
\text { webtecnologias como abordagens } \\
\text { inovadoras de aprendizagem. }\end{array}$ & $\begin{array}{l}\text { O estudo lança uma nova perspectiva sobre a } \\
\text { compreensão de como a globalização resultou } \\
\text { na internacionalização dos ambientes no } \\
\text { ensino superior. Assim, métodos de ensino } \\
\text { aplicados às webtecnologias vinculam as } \\
\text { universidades e melhoram a sua } \\
\text { democratização }\end{array}$ \\
\hline 4 & $\begin{array}{l}\text { Castel AFC. } \\
\text { et al, } 2018\end{array}$ & Educar en Revista & $\begin{array}{l}\text { La integración de las TIC's en } \\
\text { los procesos educativos y } \\
\text { organizativos }\end{array}$ & $\begin{array}{l}\text { O estudo apresenta uma revisão acerca da } \\
\text { importância das Tecnologias de } \\
\text { Informação e Comunicação com enfoque } \\
\text { na educação, sob a necessidade de ajuste } \\
\text { estratégico no contexto da universidade, } \\
\text { como um importante elemento de melhora } \\
\text { na adaptação às novas demandas na } \\
\text { aplicação de métodos de ensino } \\
\text { inovadores, sob o processo de ensino- } \\
\text { aprendizagem e o desenvolvimento } \\
\text { estratégico dessas ferramentas na formação } \\
\text { profissional }\end{array}$ & $\begin{array}{l}\text { É posto em destaque o papel das TIC's como } \\
\text { importante fator estratégico dentro do ensino } \\
\text { universitário, intimamente ligado ao modelo } \\
\text { educacional implementado na instituição de } \\
\text { ensino. Dessa forma, existe uma necessidade } \\
\text { contínua de atualização do contexto } \\
\text { educacional que vise uma análise aprofundada } \\
\text { do ambiente e do local. Centralizado no } \\
\text { aluno, é importante flexibilizar, inovar e } \\
\text { adaptar os conteúdos repassados e as } \\
\text { webtecnologias se sobressaem como um } \\
\text { recurso de fundamental importância nesse } \\
\text { quesito }\end{array}$ \\
\hline 5 & $\begin{array}{l}\text { Maldonado } \\
\text { FXJ. et al, } \\
2016\end{array}$ & $\begin{array}{l}\text { Revista Universidad } \\
\text { y Sociedad }\end{array}$ & $\begin{array}{l}\text { Educación a Distancia: una } \\
\text { necesidad para la formación de } \\
\text { los profissionales }\end{array}$ & $\begin{array}{l}\text { O artigo busca analisar os elementos } \\
\text { teóricos relativos aos estudos conceituais } \\
\text { práticos sobre educação à distancia, com a } \\
\text { utilização das TIC's, na formação inicial e } \\
\text { continuada de profissionais }\end{array}$ & $\begin{array}{l}\text { Os autores conseguiram mostrar que a } \\
\text { qualidade pedagógica e a preparação dos } \\
\text { profissionais com as TIC's, melhoram de } \\
\text { forma significativa o processo de ensino e } \\
\text { aprendizagem, além de permitir programas } \\
\text { inovadores de educação em webtecnologias } \\
\text { integrativas }\end{array}$ \\
\hline
\end{tabular}

Fonte: Autores.

O processo de ensino-aprendizagem imprime uma necessidade contínua de atualização e formação continuada, que viabilize a atenção complementar e integrativa a partir da interatividade, praticidade e autonomia da utilização dos produtos tecnológico educacionais, além de construir uma coerência significante e consistente ao contexto em que se insere. Para que as Webtecnologias cumpram seu papel como espaço integrador do indivíduo com o conhecimento, é importante que seu uso seja viabilizado a partir da remodelação multi-interdisciplinar e interprofissional com ênfase na promoção da saúde, bem como descrito por Germani, et al (2014).

Nessa perspectiva, o contexto demonstrado ao longo da formação do profissional, demanda uma ampla gama de recursos digitais que contribuam para o gerenciamento e construção do conhecimento adequado sobre as práticas clínicas e laboratoriais. Germani, et al (2014) e Jimoyan, et al (2010) destacam algumas intercorrências quanto ao uso de Webtecnologias, como recursos interativos e propõe essa deficiência à intimidade relativa do público quanto a utilização da tecnologia. Dessa forma, como já descrito, é comum que haja uma certa dificuldade na aplicação de novos modelos de ensino. Portanto, mais uma vez, se evidencia a função da instituição e do docente ou tutor, a criação de um clima colaborativo que concerne o interesse mútuo na habilidade prática do desenvolvimento do aprendizado adquirido por meio dessas ferramentas tecnológicas. 
Com base nas similaridades encontradas nos estudos de Lima, et al (2020) e Castel, et al (2018), assim como os objetivos dos estudos e métodos de abordagem empregados, foi possível verificar os benefícios que o sistema de ensino baseado em tecnologias educacionais proporcionam, abre precedentes para o acesso irrestrito à mobilidade ilimitada às experiências virtuais, principalmente com enfoque na atribuição de valores relacionados a fatores socioeconômicos que inviabilizam a globalização das cibertecnologias. Nesse viés, assim como ressaltado por Maldonado, et al (2016), o desenvolvimento de plataformas e ferramentas digitais, como as webtecnologias, podem ampliar a participação no ensino, para inclusão social e apoio à internacionalização do ensino superior. Com base nessas atribuições, sob um aspecto complementar, o Quadro 3 traz uma visão geral acerca dos artigos com enfoque na educação continuada em Citologia Clínica a fim de capacitar e formar profissionais de na área da saúde.

Quadro 3: Educação continuada em Citologia Clínica para capacitação e formação de profissionais em cursos da área da saúde.

\begin{tabular}{|c|c|c|c|c|c|}
\hline \# & Autores & Periódico & Título & Síntese & Contribuições \\
\hline 1 & $\begin{array}{l}\text { Vilaça FA. et } \\
\text { al, } 2019\end{array}$ & $\begin{array}{l}\text { Revista de Ensino em } \\
\text { Ciências e Matemática }\end{array}$ & $\begin{array}{l}\text { O Ensino de Citopatologia no } \\
\text { Contexto Universitário: um olhar } \\
\text { para a produção - publicação } \\
\text { acadêmica e sua empregabilidade } \\
\text { como ação prática de ensino }\end{array}$ & $\begin{array}{l}\text { O estudo busca identificar as } \\
\text { principais técnicas de citologia clinica } \\
\text { utilizadas no âmbito profissional, que } \\
\text { vem sido vistas como tendências nas } \\
\text { produções acadêmicas, com a } \\
\text { finalidade de estabelecer uma relação } \\
\text { multifatorial acerca do emprego } \\
\text { dessas técnicas, nas aulas práticas } \\
\text { realizadas nos ambientes de ensino } \\
\text { integrados e multidisciplinares }\end{array}$ & $\begin{array}{l}\text { É posto em discussão uma realidade } \\
\text { inerente ao ensino em citologia clínica. } \\
\text { Em virtude das dificuldades na } \\
\text { identificação morfológica e a necessidade } \\
\text { de um diagnóstico preciso, os autores } \\
\text { puderam perceber que o ensino deve-se } \\
\text { pautar em objetivos que possuam enfoque } \\
\text { na teoria e intensifique a construção das } \\
\text { habilidades individuais práticas do } \\
\text { discente que possa conduzi-lo ao } \\
\text { diagnóstico }\end{array}$ \\
\hline 2 & $\begin{array}{l}\text { Marcon MR. } \\
\text { et al. } 2010\end{array}$ & $\begin{array}{l}\text { Revista Dia a Dia } \\
\text { Educação (Ministério da } \\
\text { Educação) }\end{array}$ & $\begin{array}{l}\text { A Contextualização de Novas } \\
\text { Tecnologias em Citologia no Ensino } \\
\text { em Biologia }\end{array}$ & $\begin{array}{l}\text { O artigo traz uma abordagem voltada } \\
\text { para a necessidade inerente do Ensino } \\
\text { em Biologia como uma alternativa } \\
\text { baseada na contextualização dos } \\
\text { conteúdos, com ênfase na promoção } \\
\text { de uma aprendizagem mais } \\
\text { integradora, significativa e } \\
\text { contextualizada, mediado por novas } \\
\text { tecnologias que visem uma } \\
\text { remodelação dos parâmetros de } \\
\text { ensino }\end{array}$ & $\begin{array}{l}\text { Essa abordagem buscou realizar uma } \\
\text { comparação acerca de métodos de ensino } \\
\text { diferenciados, sob uma aprendizagem } \\
\text { mais eficiente e agradável, de modo a } \\
\text { promover mudaças na forma de } \\
\text { desenvolver os conteúdos. Foi } \\
\text { evidenciado uma interação significativa, } \\
\text { uma capacidade crítica, além de um maior } \\
\text { índice de participação e de cooperação, } \\
\text { tanto no âmbito pessoal, quanto nas } \\
\text { relações sociais }\end{array}$ \\
\hline 3 & $\begin{array}{l}\text { Tonet C. et } \\
\text { al, } 2019\end{array}$ & $\begin{array}{l}\text { Revista Brasileira de } \\
\text { Análises Clínicas }\end{array}$ & $\begin{array}{l}\text { A telecitologia na Rotina do } \\
\text { Rastreamento do Câncer de Colo } \\
\text { Uterino }\end{array}$ & $\begin{array}{l}\text { Os autores propuseram fazer uma } \\
\text { descrição sobre o uso das novas } \\
\text { telecitologias na rotina do laboratório } \\
\text { de citologia, a partir do rastreamento } \\
\text { das lesões precursoras do carcinoma } \\
\text { cervical, caracterizando-se como um } \\
\text { potencial adjuvante na formação do } \\
\text { profissional, além de promover } \\
\text { diversos incrementos para a área da } \\
\text { saúde }\end{array}$ & $\begin{array}{l}\text { O estudo trata de uma abordagem } \\
\text { inovadora da aplicabilidade da } \\
\text { telecitologia ou citologia digital no } \\
\text { laboratório de citologia, como estratégia } \\
\text { precursora para disseminação de } \\
\text { conhecimento entre estudantes e } \\
\text { profissionais, fornecendo uma plataforma } \\
\text { de discussão e possibilitando o } \\
\text { compartilhamento de espécimes } \\
\text { citológicos encontrados }\end{array}$ \\
\hline 4 & $\begin{array}{l}\text { Donnely A. } \\
2018\end{array}$ & Cancer Cytopathology & $\begin{array}{l}\text { Cytotechnology: The benefits of } \\
\text { technology in teaching }\end{array}$ & $\begin{array}{l}\text { O referido estudo busca descrever o } \\
\text { impacto das tecnologias no programa } \\
\text { de citotecnologia da Universidade de } \\
\text { Nebraska, além de mostrar como ele } \\
\text { possibilitou a educação à distância e } \\
\text { várias oportunidades oferecidas aos } \\
\text { discentes no decorrer do processo de } \\
\text { execução }\end{array}$ & $\begin{array}{l}\text { A autora evidenciou um aprimoramento } \\
\text { da experiência educacional dos discentes, } \\
\text { a expansão do programa da universidade } \\
\text { de execução, em fluxo internacional, } \\
\text { disseminação de novos projetos } \\
\text { decorrentes do programa, além de permitir } \\
\text { novas conexões, permitindo a melhora do } \\
\text { sistema educacional }\end{array}$ \\
\hline
\end{tabular}

Fonte: Autores. 
A Citologia Clinica, de uma forma assertiva, apresenta uma gama multivariada de meios inteiramente específicos que conduzem às estratégias de fomento do profissional de saúde no diagnóstico preciso de patologias. Com vistas a favorecer o entendimento acerca do diagnóstico precoce, Vilaça, et al (2019) realiza um mapeamento do conjunto de técnicas, estratégias e formas de diagnósticos mais descritos na literatura que, como aspecto fundamental na formação de profissionais capacitados, se estabelece como um fator primordial para o subsídio da sua formação com foco na melhora da habilidade e dos conhecimentos específicos. Ao considerar as técnicas desenvolvidas e a aplicabilidade em sala de aula, além de incluí-las no plano de ensino, torna-se fundamental a adesão de novas formas de ensino.

Entretanto, uma alteração nos métodos de ensino envolve vários parâmetros e diretrizes inerentes a um papel já determinado nos moldes de educação pedagógica. Foi possível ser observado no estudo de Marcon, et al (2010), que o processo de ensino aprendizagem em citologia, necessita ter uma estrutura cognitiva com foco a se distanciar do modelo vigente que, conforme descrito também por Tonet, et al (2019), carece de uma aplicabilidade e funcionalidade na rotina laboratorial. Dessa forma, essas novas perspectivas de ensino, traduzem mudanças na prática da citologia à distância, revolucionando o papel do profissional e fornecendo diversas oportunidades e incrementos das ações nas áreas. Para tanto, elaborou-se uma nuvem de palavras, que tem por função mostrar o destaque dos termos mais frequentes nos estudos (Figura 3).

Figura 3: Nuvem de Palavras dos artigos selecionados rever as numerações das figuras e todas devem ser chamadas no texto.

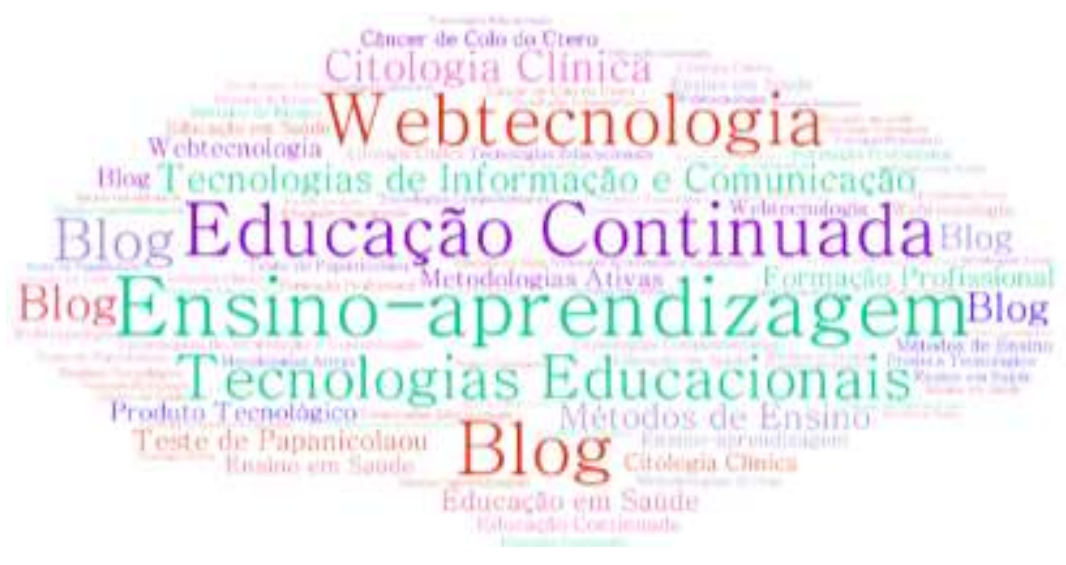

Fonte: Autores.

\section{Considerações Finais}

Todos os estudos selecionados, possuem um viés intencional de discussão com base na sua estrutura. É de confluência geral que as Instituições de Ensino Superior, desempenham um papel crítico na formação do conhecimento. Assim, apesar das modelagens estruturais de ensino estarem enraizadas na matriz docente, existe a necessidade da mudança na forma de aprendizagem, fundamentado no alcance à adaptação dos novos meios e tecnologias para o sistema de educação superior. Dessa forma, e de acordo com as tendências observadas no meio de ensino, as mudanças nos requisitos da educação e da sociedade tem sido amplamente discutida na literatura e em virtude dos aspectos inerentes às dificuldades na compreensão da citologia clínica, como já exposto, é provável que essas tecnologias descritas possam traduzir mudanças na forma como o ensino pode ser disseminado, a partir da utilização de suas ferramentas de acesso como instrumentos de inovação em ensino, possibilitando a construção e a atualização de conhecimentos. Essa revisão teve função complementar na sua elaboração e fomentou o viés para a necessidade da adesão de Webtecnologias que viabilizem o ensino em citologia. Desse modo, estudos que promovam a utilização do Blog como ferramenta educacional pode viabilizar estratégias continuadas na formação do profissional de saúde. 


\section{Referências}

Aguiar, A. C. L., et al. Blog Como Ferramenta Educacional: Contribuições Para O Processo Interdisciplinar De Educação Em Saúde. Revista Eletrônica De Comunicação, Informação E Inovação Em Saúde, [S.L.], 2018.

Alexandre, S. F. Aprendizagem E Suas Implicações No Processo Educativo. Revista De Letras Da Ueg. São Luís De Montes Belos. P. 51-60. 2010.

Amarilla Filho, P. Educação A Distância: Uma Abordagem Metodológica E Didática A Partir Dos Ambientes Virtuais. Educação Em Revista, 27(2), 41-72, 2011.

Baldissera, S. S., Et Al. Promoção Da Saúde E Prevenção Do Câncer Do Colo Uterino: Estratégias Utilizadas Pelos Enfermeiros. Research, Society And Development, [S.L.], 9(9), 2020.

Beluce, A. C., \& Oliveira, K. L. Learning Strategies Mediated By Technologies: Use And Observation Of Teachers. Paideia, 28(69), 1-10, 2018.

Bowman, M. A., et 1. Teachers' Exposure To Professional Development And The Quality Of Their Instructional Technology Use: The Mediating Role Of Teachers' Value And Ability Beliefs. Journal Of Research On Technology In Education, V. 0, N. 0, P. 1-17, 2020.

Burity, C. H., \& Cardozo, C. V. Atlas Digital De Morfologia Comparada: Uma Ferramenta Complementar Ao Ensino De Histologia E Histopatologia. Revista Uniabeu, [S.L.], 7(16), 2014.

Calumby, R. J., et al. Papiloma Vírus Humano (Hpv) E Neoplasia Cervical: Importância Da Vacinação. Brazilian Journal Of Health Review, [S.L.], 3(2), 16101628,2020

Campos, E. A., Castro, L. M., \& Cavalieri, F. E. Uma Doença Da Mulher: Experiência E Significado Do Câncer Cervical Para Mulheres Que Realizaram O Papanicolau. Interface-Comunicação, Saúde, Educação, [S.L.], 21, 385-396, 2017.

Carvalho, A. M., et al. Adesão À Vacina Hpv Entre Os Adolescentes: Revisão Integrativa. Texto \& Contexto-Enfermagem, [S.L.], V. $28,2019$.

Carvalho, P. G., et al. Trajetória Da Assistência À Saúde Da Mulher Entre O Diagnóstico E O Início Do Tratamento Do Câncer De Colo Do Útero No Brasil Emergente. Saúde Debate., [S.L.], 42(118), 687-701, 2020.

Castel, A. F. G. La Integración De Las Tic En Los Procesos Educativos Y Organizativos A Integração Das Tic Nos Processos Educacionais E Organizativos. Educar Em Revista, 34(69), 325-339, 2018

Chirelli, M. Q., Pio, D. A. M, \& Soares, M. O. M. Competência Dialógica: Avaliação Do Desempenho No Currículo Integrado. Indagatio Didactica, 8(3), $109-123,2016$.

Daroda, L. S. L. Utilização Das Tecnologias Da Informação E Comunicação Pelos Docentes De Ensino Superior Da Área Da Saúde. 2012.115 F. Dissertação (Mestrado Em Gestão E Avaliação Da Educação Pública) - Faculdade De Educação, Universidade Federal De Juiz De Fora, Juiz De Fora, 2012.

Da Costa, F. K., et al. Os Desafios Do Enfermeiro Perante A Prevenção Do Câncer Do Colo Do Útero, [S.L.], 2017.

Damiani, E., et al. Conhecimentos, Atitudes E Práticas Das Mulheres Sobre A Prevenção Do Câncer De Colo Uterino: Uma Revisão De Literatura. Brazilian Journal Of Health Review, [S.L.], 4(1), 364-381, 2021.

Domingos, G. A. Dificuldades Do Processo De Aprendizagem. 2017. P.1-29. (Monografia) - Escola Superior Aberta Do Brasil. Vila Velha. P. 5.

Donelly, Cytotechnology: The Benefits Of Technology In Teaching. Cancer Cytopathology, 126(8), 511-512.

Duarte, V. D., Et Al. Prevalência Da Infecção Pelo Papilomavírus Humano E Rastreamento Do Câncer Em Mulheres Ribeirinhas Da Amazônia Brasileira. Rev Bras Ginecol Obstet, [S.L.], 39, 50-357, 2017.

Fabrício, B. F. Repetir-Repetir Até Ficar Diferente": Práticas Descoloniais Em Um Blog Educacional. Cadernos De Linguagem E Sociedade, [S.L.], 18(2), 9$26,2017$.

Fonseca Neto, A. A., Pereira Filho, A. M., \& Silva, A. B. Conhecimento De Idosas De Uma Unidade Da Estratégia Saúde Da Família Sobre Prevenção Do Câncer Cervicouterino. Brazilian Journal Of Health Review, 3(2), 1699-1712, 2020.

Galdino, Y. L. Construção E Validação De Cartilha Educativa Para O Autocuidado Com Os Pés De Pessoas Com Diabetes. Dissertação (Mestrado Acadêmico Em Cuidados Clínicos Em Enfermagem E Saúde) - Centro De Ciências Da Universidade Estadual Do Ceará, 2014.

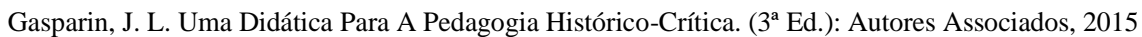

Germani, A. C. C. G. Et Al. Avaliação De Tecnologias Educacionais Digitais Para A Formação De Promotores De Saúde Em Uma Experiência Interprofissional. Jornal Brasileiro De Telessaúde, 3(1), 2014.

Ghiraldelli J, P. Didática E Teorias Educacionais. Rio De Janeiro: Dp\&A, 2000.

Hernández-Hernández, D. M., Apresa-García, T., \& Patlán-Pérez, R. M. Panorama Epidemiológico Del Cáncer Cervicouterino. Revista Médica Del Instituto Mexicano Del Seguro Social, [S.L.], 53(2), S154-S161, 2015.

Instituto Nacional De Câncer José Alencar Gomes Da Silva. Coordenação De Prevenção E Vigilância Divisão De Detecção Precoce E Apoio À Organização Da Rede. Diretrizes Brasileiras Para O Rastreamento Do Ccu - 2. Ed. Rev. Atual. - Rio De Janeiro, 2016. 
Instituto Nacional De Câncer José De Alencar Gomes Da Silva (Brasil). Nomenclatura Brasileira Para Laudos Citopatológicos Cervicais. (3 ${ }^{a}$ Ed.) Ministério Da Saúde. Rio De Janeiro: Inca, 2012.

Instituto Nacional Do Câncer. Ccu - Versão Para Profissionais De Saúde. < Https://Inca.Gov.Br/Tipos-De-Cancer/Cancer-Do-Colo-Do-Utero/Profissional-DeSaude>.

Jimoyiannis, A. Designing And Implementing An Integrated Technological Pedagogical Science Knowledge Framework For Science Teachers Professional Development. Computers And Education, 55(3), 1259-1269, 2010.

Juca Maldonado, F. X. La Educación A Distancia, Una Necesidad Para La Formación De Los Profesionales. Revista Universidad Y Sociedad, 8(1), 106-111, 2016.

Lacanallo, Et Al. Métodos De Ensino E De Aprendizagem: Uma Análise Histórica Educacional Do Trabalho Didático, E. 2, V. 12, Ed. Pensamento E Linguagem, 2019 .

Lakatos, E., \& Marconi, M. Fundamentos De Metodologia Científica. São Paulo: Atlas, 2001.

Lima, Et Al. Education, H. Plataformas Digitais De Aprendizagem : Uma Revisão Integrativa Para Apoiar A Internacionalização Do Ensino Superior. P. 1-18, [S.D.].

Lima, M. R. Blog Educacional E Dispositivos Móveis: Uma Proposta De Intervenção Em Cursos Técnicos Integrados Ao Ensino Médio Na Modalidade De Educação De Jovens E Adultos. [S.L.], 2019.

Malta Rocha, L. M. B., Ferreira, A. M. V., \& Fonseca Vieira, M. De L. Blog Educacional: Descritores No Mestrado Profissional Em Ensino Na Saúde (Mpes). Revista Tecnologia E Sociedade, 15(37), 137-146, 2019.

Marcon, M. R., \& Stange, C. E. B. A Contextualização De Novas Tecnologias Em Citologia No Ensino Em Biologia. Revista Dia A Dia Educação (Ministério Da Educação), 1, 121-145, 2010.

Ministério Da Saúde. Instituto Nacional De Câncer. Coordenação Geral De Ações Estratégicas. Divisão De Apoio À Rede De Atenção Oncológica. Diretrizes Brasileiras Para O Rastreamento Do Câncer Do Colo Do Útero. Rio De Janeiro: Inca; 2012.

Mortimer, E. F., Chagas, A. N., \& Alvarenga, V. T. Linguagem Científica Versus Linguagem Comum Nas Respostas Escritas. Investigações Em Ensino De Ciências, Porto Alegre, 3(1), 1998.

Modelski, D., Giraffa, L. M. M., \& Casartelli, A. De O. Tecnologias Digitais, Formação Docente E Práticas Pedagógicas. Educação E Pesquisa, 45, 1-17, 2019.

Melo, E. M., Et Al. Câncer Cervico-Uterino: Conhecimento, Atitude E Prática Sobre O Exame De Prevenção. Revista Brasileira De Enfermagem, [S.L.], 72, 25-31, 2019.

Ministério Da Saúde. Arquivos Da Secretária De Vigilância Em Saúde, Dasis; Cgiae. Sistema De Informação Sobre Mortalidade, 2021.

Moura, L. D., Codeço, C. T., \& Luz, P. M. Cobertura Da Vacina Papilomavírus Humano (Hpv) No Brasil: Heterogeneidade Espacial E Entre Coortes Etárias. Revista Brasileira De Epidemiologia, [S.L.], 24, 2020.

Nayar, R., \& Wilbur, D. C. The Pap Test And Bethesda 2014. Acta Cytol., [S.L.], 59(2), 121-32, 2015

Nietsche, E. A. Tecnologias Educacionais, Assistenciais E Gerenciais: Uma Reflexão A Partir Da Concepção Dos Docentes De Enfermagem. Revista LatinoAmericana De Enfermagem, [S.L.], 13(3), 344-352, 2005.

Palangana, I. C. Desenvolvimento E Aprendizagem Em Piaget E Vigotsky (A Relevância Do Social). Editora: Plexus - (2ª Edição). 1998.

Pinheiro, D. N. Fatores Interferentes Nas Estratégias De Controle Do Câncer Do Colo Do Útero Com Ênfase Na Infecção Pelo Hpv. 2014. 87 F. Tese (Doutorado) - Universidade Federal Do Pará, Núcleo De Medicina Tropical, Programa De Pós-Graduação Em Doenças Tropicais, Belém, 2014.

Pereira, I. D. Análise Descritiva Da Cobertura Da Vacina Hpv Quadrivalente No Brasil, Entre 2016 E 2017, 2018.

Peuker, A. C., Et Al. Construção De Um Material Educativo Para A Prevenção Do Câncer De Colo Do Útero. Estudos Interdisciplinares Em Psicologia, [S.L.], $8(2), 146-160,2017$.

Rodrigues. A. K. Análise Do Perfil De Mulheres Atendidas Pela Sesma Diagnosticadas Com Ascus No Período De 2013 A 2015 No Estado Do Pará. Revista Conexão Uepg, [S.L.], 3(2), 2017.

Rodrigues, A. L., Et Al. Cobertura Vacinal Do Hpv: Uma Análise Sobre Fatores Que Implicam Na Baixa Adesão À Vacina. Revista Transformar, [S.L.], 14(1), 560-574, 2020.

Santo, S. A. C. Do E., Moura, G. C. De. S., Joelma, T. Da. O Uso Da Tecnologia Na Educação: Perspectivas E Entraves. Revista Científica Multidisciplinar Núcleo Do Conhecimento. Ano 05, Ed. 01, Vol. 04, Pp. 31-45. 2020.

Santos, S. C. S. C., \& Kaulfuss, M. A. Processo De Ensino Aprendizagem No Contexto Escolar : Dificuldades E Transtornos De Aprendizagem. Editora Plexus, 1997 [S.D.].

Shaffer, D. W., Nash, P., \& Ruis, A. R. Technology And The New Professionalization Of Teaching. Teachers College Record, 117(12), 2015.

Sanches, B. E. Caracterização Sociodemográfica De Mulheres Do Programa De Prevenção Do Câncer De Colo Do Útero Em Um Centro De Saúde Escola. Pesquisa Em Saúde, [S.L.], (14), P. 64-70, 2015. 
Research, Society and Development, v. 10, n. 10, e87101018796, 2021

(CC BY 4.0) | ISSN 2525-3409 | DOI: http://dx.doi.org/10.33448/rsd-v10i10.18796

Sanches, B. E., Et Al. Infecção Por Papilomavírus Humano E Lesões Precursoras Do Câncer Cérvico Uterino Em Ribeirinhas Da Amazônia: Avaliação Da Relação Com Marcadores De Estresse Oxidativo. Revista Eistein, [S.L.], 2018.

Silveira, A. C. M. Da. (Org.). Divulgação Científica E Tecnologias De Informação E Comunicação. Santa Maria: Facos, Ufsm, 2003

Stelet, B. P., Et Al. Reflective Portfolio: Philosophical Contributions To A Narrative Praxis In Medical Education. Interface (Botucatu), 21(60), 165-176, 2017.

Santos, G. F. Perfil Sócio Demográfico Epidemiológico Reprodutivo Alimentar De Mulheres Ribeirinhas Com Lesões Precursoras Do Câncer De Colo Uterino, No Estado Do Pará. 2017.97f. Dissertação(Mestrado Em Em Doenças Tropicais) - Universidade Federal Do Pará, Núcleo De Medicina Tropical, Belém, 2017.

Santos, J. S., \& Lacerda, N. A. A Linguagem Dos Alunos Na Escrita Colaborativa Em Blog Educacional Para O Ensino-Aprendizagem De Língua Portuguesa. Letras, Linguística E Artes: Perspectivas Críticas E Teóricas, [S.L.], V. 108, 2019.

Siqueira, G. S., Et Al. Citopatologia Como Prevenção Do Câncer Do Colo Uterino. Cadernos De Graduação - Ciências Biológicas E Da Saúde Unit, Aracaju, 2(1), 37-49, 2014.

Solomon, D., Nayar.R., \& Wilbur. E. El Sistema Bethesda Para Informar La Citologia Cervical. Definiciones, Critérios Y Notas Aclatorias. 3. Ed. 2017.

Tacca, M. C. V. R., \& Branco, A. U. Processos De Significação Na Relação Professor-Alunos: Uma Perpsctiva Sociocultural Construtivista.Estudos De Psicologia, 13(1), 39-48, 2018.

Viana, G. M. Atlas Digital No Auxílio E Aprendizado De Histologia E De Citologia. Fórum De Ensino, Pesquisa E Extensão, 2017.

Vieira, E. S., Belo, P. A., \& Freire, V. C. Possibilidade De Utilização Do Blog Como Ferramenta Educacional. Práticas Educativas, Memórias E OralidadesRev. Pemo, [S.L.], 2(2), 2020.

Vilaça, F. A., Siqueira, A. C., \& Frenedozo, R. C. O Ensino De Citopatologia No Contexto Universitário: Um Olhar Para A Produção/Publicação Acadêmica E Sua Empregabilidade Como Ação Prática De Ensino. Revista De Ensino De Ciências E Matemática, 10(3), 168-187, 2019.

Zamunaro, A. M. B. R. A Prática De Ensino De Ciências E Biologia E Seu Papel Na Formação De Professores. Bauru: Universidade Estadual Paulista, P. 236, 2006.

Zapponi, A. L., \& Melo, E. C. Distribuição Da Mortalidade Por Câncer De Mama E De Colo De Útero Segundo Regiões Brasileiras. Rev. Enferm. Uerj, [S.L.], P. 628-631, 2010. 